\title{
Feeling the future: prospects for a theory of implicit prospection
}

\author{
Philip Gerrans • David Sander
}

Received: 15 March 2013/ Accepted: 28 November 2013/Published online: 8 December 2013

(C) Springer Science+Business Media Dordrecht 2013

\begin{abstract}
Mental time travel refers to the ability of an organism to project herself backward and forward in time, using episodic memory and imagination to simulate past and future experiences. The evolution of mental time travel gives humans a unique capacity for prospection: the ability to pre-experience the future. Discussions of mental time travel treat it as an instance of explicit prospection. We argue that implicit simulations of past and future experience can also be used as a way of gaining information about the future to shape preferences and guide behaviour.
\end{abstract}

Keywords Mental time travel - Somatic marker hypothesis · Implicit processes · Iowa gambling task · Prospection

In mental time travel we review past and preview future experiences. This is a crucial cognitive adaptation for humans, tied to the evolution of the prefrontal cortex, which allows us to simulate possible futures when deliberating. The concept of mental time travel understood as a distinct cognitive process with a specialised neural substrate is now supported by convergent evidence from the neurosciences of memory, imagination, planning and decision-making. As Schacter et al. put it: "the medial temporal lobe system which has long been considered to be crucial for remembering the past might actually gain adaptive value through its ability to

\footnotetext{
P. Gerrans $(\bowtie) \cdot$ D. Sander

Swiss Centre for Affective Sciences, University of Geneva, Geneva, Switzerland

e-mail: philip.gerrans@adelaide.edu.au

P. Gerrans

Department of Philosophy, University of Adelaide, Adelaide, Australia

D. Sander

Department of Psychology, University of Geneva, Geneva, Switzerland
} 
provide details that serve as building blocks of future event simulations" (Schacter et al. 2007, p. 659; See also Addis et al. 2007; Schacter and Addis 2007b Buckner and Carroll 2007; Busby and Suddendorf 2005; Hassabis and Maguire 2007; Spreng et al. 2009; Suddendorf and Corballis 2007, Suddendorf et al. 2009. See Szpunar 2010 for a comprehensive review of the hypothesis that episodic memory and imagination, are both constructive processes which depend on the same neural systems).

The extent to which mental time travel is unique to humans is controversial. It is tested in scenarios that involve episodic memory and prospection such as perceptual recollection, planning and decision-making, or detected in situations in which the mind reverts to daydreaming or mindwandering (Christoff et al. 2009; Greicius et al. 2003; Mason et al. 2007). Typically in such cases human subjects can describe their experience and the content of the imagery involved. Not only that but convergent evidence from neuroscience supports the idea that what the brain is doing in these cases is simulating episodes of personal experience.

When, however, we turn our attention to highly intelligent primates and birds and to pre or barely verbal infants, determining the nature and content of cognitive processes and experiences is not straightforward. Consider, for example, Santino the chimpanzee who hoards rocks to throw at visitors to his enclosure. Is he remembering previous episodes of harassment and imagining banishing future voyeurs? What is going in the mind of chimpanzees who will not retrieve food while observed by a dominant male (Call and Tomasello 2008)? Scrub jays cache food for winter and will re-cache it if observed by other jays (Clayton and Dickinson 1998; Clayton et al. 2003; Dally et al. 2006) Are they recalling previous thefts and anticipating a hungry future? These are instances of intelligent future-directed behaviour that seem to involve the representation of information about past and future contingencies unavailable in the current experience. Purely stimulus-bound creatures could not produce these behaviours. However the intelligence of the behaviour could be a product of a combination of associative learning, semantic memory and complex environmental scaffolding (Brass and Heyes 2005; Heyes 1998, 2010; Shettleworth 2010).

Clearly, if scrub jays and chimpanzees are engaged in mental time travel then their behaviour is easily explained. But the consensus is that they are not mental time travellers. Their behaviour across a range of domains and evidence about their neurocognitive architectures do not support the idea that they are capable of explicit prospection: the simulation of a future experience to guide decision-making (Roberts and Feeney 2009). Of course their capacity for mental time travel may be limited to specific ecological contexts: but that creates another puzzle. Why would a capacity (simulation) that is essentially stimulus independent and confers such adaptive advantages be used in such a restricted way?

Other interesting and difficult cases are raised by infant and toddler cognition. Pre schoolers are capable of future-directed cognition in limited contexts but whether they are in fact able to sample past and future experiences in imagination and memory is a difficult question. There is a lot of evidence to suggest that the preschool child is not able to use mental time travel to control her behaviour until her prefrontal cortex matures sufficiently to enable explicit simulation of experience 
under a degree of executive control (Fair et al. 2008; Garon et al. 2008; Leslie 1994; Zelazo and Frye 1998).

Our aim in this paper is to argue that there are forms of prospection that can be guided by implicit simulation of information represented in past and future experience. Those implicit simulations are not fully under executive control (as in adult humans who can, for example, imaginatively rehearse the nausea produced by the lurching deck and the stench of overflowing toilets when deciding to fly rather than travel by ferry) but they provide a form of cognitive control which may well be a precursor of fully fledged mental time travel. Our aim is to make conceptual space for the possibility of implicit prospection and show that it is at least consistent with some of the neurocognitive and behavioural evidence. We shall rely on some evidence that many (not all) cognitive processes involving the construction of explicit representations depend on prior stages of implicit processing which represent information at coarser levels of grain in order to enable rapid cognitive and behavioural response. Thus, for example, explicitly recognising a face or place is the final stage in a process that initially involves implicit recognition. Our claim is that these "early" implicit forms of representation can play a similar role in decision making to the role played by explicit representations in mental time travel.

In general we endorse the strategy of not postulating additional representational complexity where rich environmental structure and well-established cognitive capacities can account for behavioural complexity. For example creatures (like toddlers, chimpanzees and scrub jays) who possess semantic knowledge (of facts) and are capable of affectively-scaffolded associative learning may, in suitably structured environments show very intelligent future directed behaviour. It could be, for example, that scrub jays flexible cache-ing behaviour is an associatively learnt response to conditions of scarcity and population density (thanks to an anonymous referee for the suggestion). And toddlers may know that a new baby will be arriving by Christmas time put a present under the tree for her even if they cannot imagine the nature of siblinghood.

At present one might think that the options for explaining future directed behaviour consisted in

(i) associative learning plus a rich environment

(ii) semantic knowledge

(iii) combination of (i) and (ii)

(iv) fully-fledged mental time travel in which episodes of experience are simulated

(v) explicit reasoning

Clearly (iv) and (v) represent distinctly human abilities not present in infants but certainly present in primary schoolers.

We note that, given that mental time travel exists as a sophisticated cognitive adaptation, it would be surprising if it did not have one or more less sophisticated precursors, ontogenetically or phylogenetically. Equally, given that explicit reasoning does not arrive as an all or nothing ability but has a developmental pathway it also has some precursor stages. Our idea is that a combination of implicit representation plus less than fully-fledged executive control may provide a capacity for implicit prospection. We do not see ourselves as postulating additional cognitive 
mechanisms or processes to account for behaviour that can be accounted for in other ways. Rather we see ourselves as directing attention to a role for implicit cognitive processes that is consistent with evidence about cognitive architecture and performance. We suggest that implicit mental time travel may be an instance of the deployment of existing capacities for implicit representation and developing capacity for executive control to confer cognitive advantages. Our thought is that if the transition from (iii) to (iv) and (v) is not all or nothing then there may be behaviours that represent a kind of intermediate developmental stage.

Episodic memory and controlled imagination arrive around the age of four as part of a suite of executive capacities that provide a capacity for explicit simulation and a degree of foresight. The capacities of pre-school children for explicit deliberation and controlled simulation are quite limited. This has been advanced as one reason why infants younger than about three and a half cannot pass the false belief test, which requires them to predict where another person will look for an item whose location has been changed since they last saw it. Interestingly however in "violation of expectation" and "anticipatory looking" paradigms children as young as 15 months have passed version of the false belief test (Ruffman 2000). All theorists agree that these tasks involve implicit representation, and many have interpreted these cases as evidence of implicit representation of the concept of false belief. Debate continues. For our purposes it is important to note only that that these children are asked to predict the future based on previous experience and that the representation of the right location is stimulus independent.

Of course it may be the case that the solution here actually involves (i) (ii) or (iii) but our point is that it may also involve a precursor stage of the development of (iv) and (v). That is to say the child implicitly remembers where the object was and uses that representation to guide predictive behaviour. Executive control at this stage may consist only in some top down inhibition of stimulus-driven behavioural output while implicit representations of alternatives are rehearsed before neural activity settles into a stable pattern that determines output behaviour (Koechlin and Summerfield 2007). We see instances of this kind of very limited executive control in cases where response times in decision-making tasks are affected by the presentation of alternatives with different affective valence. Often the presentation of alternatives produces an interference effect, a different pattern of neural activity and slower response time. It is as though alternatives are being tacitly rehearsed before the choice or decision is made even though the subject cannot explicitly represent the decision making process (Bowman et al. 2005). If this is the case it would not be surprising if the lack of executive capacity meant that implicit and explicit performance on false belief tasks is not consistent in toddlers. Perhaps toddlers cannot recall, construct, maintain and manipulate an explicit representation of the relevant information under voluntary control (Stuss and Alexander 2000). The information is, however, retained, and an implicit representation that guides reflexive behaviour can be elicited under the right conditions.

If this is the case it would not be surprising if the lack of executive capacity meant that performance was very limited. Perhaps toddlers cannot recall, construct, maintain and manipulate an explicit representation of the relevant information under 
voluntary control. The information is, however, retained, and an implicit representation that guides reflexive behaviour can be elicited under the right conditions.

Such a precursor stage need not actually be a distinct cognitive capacity but might consist in the way implicit capacities are used in concert with developing capacities for executive control (Zelazo and Frye 1998). Often cognitive development consists in being able to make explicit, under full executive control, information, which was formerly implicit, only partially accessible and controllable. Mapping the concept of relative magnitude to the counting series in order to grasp the concept of number is an example. Toddlers are not bad at estimating "bigger than" and counting "one"; "two"... "many" and they can chant the counting series, but putting the concepts together by representing something like an infinite number line of regularly spaced integers is a breakthrough (Sarnecka and Carey 2008; Delazer and Benke 1999). The point is that before they do that they may exhibit some "intermediate" stage arithmetical behaviour which represents, not a maturation of a distinct cognitive adaptation for "intermediate arithmetic", but a stage in the culturally scaffolded deployment of existing representational capacities en route to an explicit representation of numbers Sterelny 2011). Part of this stage might consist in good performance on some counting or estimation tasks accompanied by inability to fully articulate and control the behaviour via explicit representation. Just as the pre-schooler does well at implicit false belief tests and poorly at explicit ones.

Such cases should not necessarily be taken as evidence that implicit and explicit representations of a domain are distinct cognitive capacities (Davies 1987) with entirely discrete neural substrates, but that explicitness and cognitive control come in degrees for some conceptual domains (Maia and McClelland 2004, 2005; Degonda et al. 2005) We give examples in later sections, which we take to be uncontroversial. We add the idea that the implicit representations in question can sometimes accessed in the service of future-directed cognition, allowing the subject to simulate alternative responses in order to guide behaviour. Initially we concentrate on a hypothetical case of prospection by a prosopagnosic patient. We discuss alternative interpretations of this case as a way of clarifying the issues. Our discussion of these alternatives leads to discussion of the relationship between implicit and explicit simulation in another well-known case, the Iowa gambling task (IGT). We argue that the IGT may have been misinterpreted as evidence for a dual process architecture of decision making in which implicit and explicit processing are conceived of as distinct cognitive processes.

\section{Implicit recognition: the case of faces}

Prosopagnosia comes in different forms but the relevant one for us is one in which a subject can recognise a face qua face and recognise facial features but is unable to determine whether the face, even of a relative, is familiar to her. Such patients will produce identical responses when presented with both familiar and unfamiliar faces. They have associative prosopagnosia in which they are unable to overtly (explicitly) recognise familiar faces. Nonetheless in some cases lack of explicit recognition is accompanied by implicit recognition (called covert recognition in the face recognition 
literature). Covert recognition is evidenced by interference and priming effects (the presence of a familiar face changes behaviour even though subjects are not aware that they have seen the face before) and skin conductance response (SCR indicative of autonomic activity prompted by recognition) for familiar faces (Bauer 1984, 1986; Breen et al. 2000; 2001; De Haan et al. 1987a, 1987b; Young 1994; Tranel and Damasio 1988a, 1998b; Tranel et al. 1995; Schweinberger and Burton 2003).

Consequently as Young puts it, "it is inadequate to think of it (prosopagnosia) as simply involving loss of recognitional mechanisms. Instead, at least some degree of recognition does take place. What has been lost is awareness of recognition." (quoted by Schweinberger and Burton 2003, p. 284).

Of course what is retained is as important as what is lost in these cases. What is retained is not simply a conditioned response to familiar faces but the early information processing on which such responses depend. In other words, the implicit representation of faces and their consequent emotional significance. (Breen et al. 2000, 2001). Importantly this affective information surfaces in awareness as a "feeling" rather than a fully explicit verbalisable thought. It thus represents a form of awareness which is less than fully explicit but which is not absent from consciousness. Thus SCR to familiar faces should not be interpreted as evidence of non representational "mere exposure" effects or preferences for familiar stimuli established by Zajonc. The intact responses of prosopagnosics to familiar faces depend on the ability to represent faces, or features of faces as familiar. This fact about the relationship between affective feeling and explicit cognition has led theorists such Hauser (2006) to describe the role of the ventromedial cortex in decision making as "trafficking" information from lower level implicit affective processes to associate it with explicitly represented information.

Although the architecture of the face recognition system is not completely characterised the following model (see Fig. 1) explains the phenomena we have described. It describes a series of processes, starting with construction of a structural description of a face derived from visual input and progressively integrating information about familiarity and identity, which culminate in explicit recognition. Importantly amygdala activation, which produces SCR, is a consequence of implicit recognition in the ventral processing stream.

Thus explicit representation of facial familiarity depends on the prior construction of implicit representations.

So far we have sought only to establish that there are implicit representations of faces, facial familiarity and emotional significance of faces.

The next stage of our argument is to show, in principle, that a subject could implicitly simulate recognising and responding affectively to a familiar face. If such simulations could be evoked and deployed in the service of future directed actions the process would be an instance of implicit prospection.

\section{Implicit prospection}

Imagine an overtly associative prosopagnosic patient being asked to select dining companions from a set of photographs of familiar and unfamiliar people. A normal 


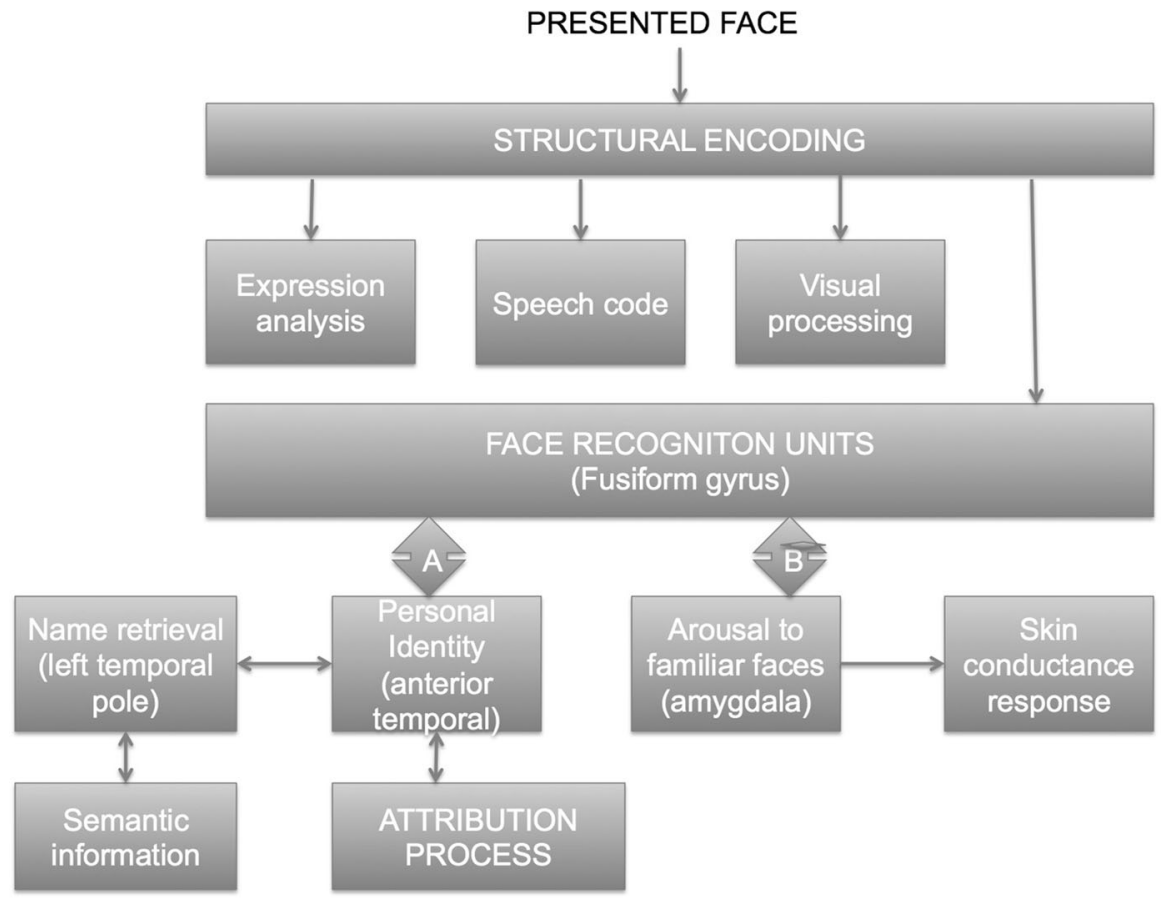

Fig. 1 The face recognition system

person could solve this problem by imagining or recalling sitting next to particular people and sampling the emotional response. She may of course solve it in other ways such as activating a conditioned response to a name but assume that she spends some time visualising alternative possibilities and chooses on the basis of feelings evoked in the process.

Someone with associative prosopagnosia cannot explicitly represent familiarity or affective significance of faces. The only resources available to our prosopagnosic to solve her problem of choosing dining companions for next week are implicit representations of familiarity and associated affective response.

We think that that nonetheless the prosopagnosic would have some strong preferences. Familiar to unfamiliar, and friends to enemies for example. And these preferences would align with her responses to familiar people prior to her injury. In other words her anticipation of prospects for an enjoyable dinner would be based on retrieving implicit information from the past in order to simulate the future. The point is not just that she has an implicit response (behavioural or affective) to particular people but that she simulates those responses as part of a future directed decision making process.

We think that this is sufficient to qualify as implicit prospection.

There are different lines of objections to this interpretation. The first is that the patient's preferences are not evidence of implicit processing. They are conditioned responses akin to mere exposure effects or affective responses acquired through 
associative learning. In the former case the response is not affective, in the second affective but in each case the representational demands are minimal. Our reply to this objection is that this is in fact why we chose the example of the prosopagnosic. The model of face recognition which structures research in the area depends on the idea that familiarity of faces is computed in order to produce the kind of behavioural effects we are interested in. In other words the relevant information is implicitly represented. The same patterns of activation in the FRU which produce priming and interference are required to produce preferences. At the very least the subject must be able to implicitly recognise the face.

A deeper objection is that while the patient does make use of implicit representation of faces the process is not one of implicit simulation. If this true then the decision making in question is not sufficiently analogous to mental time travel to count as implicit prospection.

\section{The Iowa gambling task. associative learning or implicit prospection?}

We note however that this second objection runs counter to standard interpretations of important experiments on future directed cognition. Perhaps the most famous of these is the performance of patients with damage to the ventromedial prefrontal cortex on the IGT. In the IGT subjects draw cards that prescribe a financial reward or punishment (lose or gain money) from four decks. Each deck has a different pattern of gains and losses: the "punishment schedule". Typically after a learning period subject's choices come to reflect the punishment schedules even before they are able to explicitly articulate those schedules. (Damasio et al. 1991; Bechara et al. 1996; Dunn et al. 2006).

The salient points are:

(i) normal subjects preferences align with punishment schedules

(ii) normal subjects exhibit "anticipatory SCR" for adverse decks

(iii) patients with ventromedial prefrontal damage do not adjust their choices to reflect the punishment schedules

(iv) even after they can explicitly represent the schedules

(v) patients with ventromedial prefrontal damage do not exhibit anticipatory SCR

The somatic marker hypothesis was proposed to explain why ventromedial patients who show a deficit in personal decision-making do not seem to exhibit an anticipatory $S C R$ prior to explicit decision-making. The basic idea is that idea is that the emotional significance of stimuli is implicitly represented and guides choice in the absence of explicit representation. Somatic markers are a form of implicit emotional memory postulated to allow one to decide advantageously before (explicitly) knowing the advantageous strategy (Damasio et al. 1991).

Ventromedial patients in contrast cannot generate aversive SCRs and do not seem to learn from experience. Their choices remain unconstrained even after they can explicitly articulate the punishment schedule for each deck.

The crucial point is that the SCR's in question are interpreted as "anticipatory". That is as guides to future action. This is why these studies are recruited part of the 
prospection literature. They are evidence that decision making depends on simulation of future affective response by reconstructing a previous one.

If the IGT is an instance of prospection then our example of a prosopagnosic choosing among dinner companions is equally an instance of prospection.

We note that someone might insist that both the IGT and our example are not really prospective since the stimulus is present. In that case we suggest changing the example to imagining having dinner or drawing from the relevant deck. The example then becomes "Imagine dining with X, Y or Z, or drawing from deck A, B or C. What is your preference?". If the somatic marker hypothesis is correct then a ventromedial patient would have no preferences because she could not associate an affective response with the symbolic representation of the options (X,Y,Z; A,B,C). This is correct but why?

We think that the somatic marker hypothesis suggests that the problem is solved by triggering sufficient of the early implicit responses involved to generate an autonomic response that then structures preferences. Of course the person may not be able to make the preference structure fully explicit because she has incomplete access to the representations which generated it. In that sense she is in a similar situation to the prosopagnosic who has preferences for faces she cannot articulate.

Why should we not call this a case of implicit prospection? Cleary it is not fully fledged mental time travel because all the relevant information is not explicitly represented. But relevant implicit information is represented. Furthermore the case is not simply a case of associative learning or mere exposure effects because the stimulus is no longer present. The autonomic response is modulated by the representation of emotionally salient information (Crone et al. 2004). It is true that the decision making process is triggered by a verbal representation but the very fact that a verbal representation can serve as a trigger suggests a degree of stimulus independence. After all, the subject was conditioned by initial experience, not the word or name.

A study by Tomb et al. (2002) supports the idea that explicitness comes in degrees. Tomb et al. divided 200 participants in the IGT into high, moderate and low performing groups based on results. Interestingly, only in the high performing group were anticipatory SCRs as predicted by the somatic marker hypothesis. This suggests that some subjects (the moderate group) can learn the schedule without developing anticipatory SCR. If this is the case, and explicit knowledge is not the cause, then implicit information must be playing a crucial role. It is the equivalent of the prospagnosic being able to choose dinner companions without being able to give a rationale.

Tomb and collaborators take this case to support the idea that progressive acquisition of explicit knowledge is the basis of success in the IGT i.e. that the difference between good and moderate performers consists in the degree of explicit knowledge. We do not have to endorse that conclusions to note that their explanation suggests that implicit representation precedes explicit and that SCR depends on implicit representation (see Dunn et al. 2006, p. 250). By the time the subjects can explicitly represent the schedule their implicit representations and affective responses are consistent. 
The upshot for the theory of implicit prospection is that if an organism can generate and use those implicit representations in the absence of the stimulus she is effectively remembering or imagining the represented object. Furthermore if she can do so in the service of future-oriented action she is in effect performing an act of implicit prospection.

\section{Conclusion}

We have not argued that implicit prospection exists as a distinct cognitive capacity like language or memory. Rather, like its big brother mental time travel, it consists of the ability to recruit pre-existing cognitive capacities in the service of future directed cognition. Mental time travel recruits episodic memory and imagination, explicit forms of representation, under voluntary control, to assist with deliberation. Pascal Boyer has suggested that this enables us to avoid catastrophic discounting of the future by projecting ourselves forward in time. We can feel the future as well as think about it by rehearsing future episodes of our autobiography. As he points out, people with ventromedial damage who are hostage to their stimulus driven impulses effectively "discount the future" but the way they do so is interesting. They do have propositional knowledge about the future, they know the punishment schedules in the IGT for example, but that knowledge does not affect their behaviour because it cannot override the motivational salience of the current stimulus. In order to escape the present we need to be able, not just to have semantic knowledge about future contingencies, but to sample the relevant experiences by rehearsing salient affective features of previous encounters.

There is another sense then in which the ventromedial patients are not discounting the future at all because they are not representing the present and the future in the same way: as affectively and motivationally salient. Mental time travel allows the costs of alternative actions to be calculated in the same affective currency by allowing the future to be "felt" as well as thought.

The ability to feel alternative futures however may not be something that always involves the ability to fully rehearse all aspects of the relevant experience under executive control. It might consist only in sustaining a period of indecision sufficient to allow processes of implicit representation to determine a preference ranking. The point is just that as long as all the options are not currently perceptible then such a process cannot be described as a merely conditioned response.

What would an implicit version of mental time travel look like? A creature who could do it would be able to represent future contingencies and develop preferences about them in virtue of activating implicit representations of those contingencies and their affective consequences. The creature would have adaptive future directed behaviour in virtue of information not present in the current environment. The behaviour would have to depend on something more cognitively sophisticated than associative learning and semantic memory.

It these requirements on implicit prospection seem weak we think that this is a virtue of the account. Phylogenetically and ontogenetically it is highly unlikely that the ability to project a rich explicit representation of a self in time arrives fully 
formed. The idea that mental time travel is preceded by some form of implicit prospection fits with the idea that explicitness and the ability to make use of it comes in degrees.

\section{References}

Addis DR, Wong AT, Schacter DL (2007) Remembering the past and imagining the future: common and distinct neural substrates during event construction and elaboration. Neuropsychologia 45(7):1363-1377

Bauer RM (1984) Autonomic recognition of names and faces in prosopagnosia: a neuropsychological application of the guilty knowledge test. Neuropsychologia 22:457-469

Bauer RM (1986) The cognitive psychophysiology of prosopagnosia. In: Ellis HD, Jeeves MA, Newcombe F, Young A (eds) Aspects of face processing. Martinus Nijhoff, Dordrecht, pp 253-267

Bechara A, Tranel D, Damasio H, Damasio AR (1996) Failure to respond autonomically to anticipated future outcomes following damage to prefrontal cortex. Cereb Cortex 6(2):215-225

Bowman $\mathrm{CH}$, Evans CE, Turnbull $\mathrm{OH}$ (2005) Artificial time constraints on the Iowa gambling task: the effects on behavioural performance and subjective experience. Brain Cognit 57(1):21-25

Brass M, Heyes C (2005) Imitation: is cognitive neuroscience solving the correspondence problem? Trends Cognit Sci 9(10):489-495

Breen N, Caine D, Coltheart M, Hendy J, Roberts C (2000) Towards an understanding of delusions of misidentification. Mind Lang 15:74-110

Breen N, Coltheart M, Caine D (2001) A two-way window on face recognition. Trends Cognit Sci 5:234-235

Buckner RL, Carroll DC (2007) Self-projection and the brain. Trends Cognit Sci 11(2):49-57

Busby J, Suddendorf T (2005) Recalling yesterday and predicting tomorrow. Cognit Dev 20:362-372

Call J, Tomasello M (2008) Does the chimpanzee have a theory of mind? 30 years later. Trends Cognit Sci 12(5):187-192

Christoff K, Gordon AM, Smallwood J, Smith R, Schooler JW (2009) Experience sampling during fMRI reveals default network and executive system contributions to mind wandering. Proc Natl Acad Sci 106(21):8719-8724

Clayton NS, Dickinson A (1998) Episodic-like memory during cache recovery by scrub jays. Nature 395(6699):272-274

Clayton NS, Bussey TJ, Dickinson A (2003) Can animals recall the past and plan for the future? Nat Rev Neurosci 4:685-691

Crone EA, Somsen RJ, Van Beek B, Van Der Molen MW (2004) Heart rate and skin conductance analysis of antecendents and consequences of decision making. Psychophysiology 41(4):531-540

Dally JM, Emery NJ, Clayton NS (2006) Food-caching western scrub-jays keep track of who was watching when. Science 312(5780):1662-1665

Damasio AR, Tranel D, Damasio H (1991) Somatic markers and the guidance of behaviour: theory and preliminary testing. In: Levin HS, Eisenberg HM, Benton AL (eds) Frontal lobe function and dysfunction. Oxford University Press, New York, pp 217-229

Davies M (1987) Tacit knowledge and semantic theory mind. Mind 96:441-462

De Haan EHF, Young A, Newcombe F (1987a) Faces interfere with name classification in a prosopagnosic patient. Cortex 2:309-316

De Haan EHF, Young A, Newcombe F (1987b) Face recognition without awareness. Cognit Neuropsychol 4:385-415

Degonda N, Mondadori CR, Bosshardt S, Schmidt CF, Boesiger P, Nitsch RM, Hock C, Henke K (2005) Implicit associative learning engages the hippocampus and interacts with explicit associative learning. Neuron 46(3):505-520

Delazer M, Benke T (1999) Arithmetic reasoning and implicit memory. Brain Cognit 40:94-97

Dunn BD, Dalgleish T, Lawrence AD (2006) The somatic marker hypothesis: a critical evaluation. Neurosci Biobehav Rev 239-271(30):239-271

Fair DA, Cohen AL, Dosenbach NU, Church JA, Miezin FM, Barch DM, Schlaggar BL (2008) The maturing architecture of the brain's default network. Proc Natl Acad Sci 105(10):4028-4032 
Garon N, Bryson SE, Smith IM (2008) Executive function in preschoolers: a review using an integrative framework. Psychol Bull 134(1):31

Greicius MD, Krasnow B, Reiss AL, Menon V (2003) Functional connectivity in the resting brain: a network analysis of the default mode hypothesis. Proc Natl Acad Sci 100(1):253-258

Hassabis D, Maguire EA (2007) Deconstructing episodic memory with construction. Trends Cognit Sci 11(7):299-306

Hauser MD (2006) The liver and the moral organ. Soc Cognit Affect Neurosci 1(3):214-220

Heyes CM (1998) Theory of mind in nonhuman primates. Behav Brain Sci 21(1):101-114

Heyes C (2010) Where do mirror neurons come from? Neurosci Biobehav Rev 34(4):575-583

Koechlin E, Summerfield C (2007) An information theoretical approach to prefrontal executive function. Trends Cognit Sci 11(6):229-235

Leslie AM (1994) Pretending and believing: issues in the theory of ToMM. Cognition 50:211-238

Maia TV, McClelland JL (2004) A reexamination of the evidence for the somatic marker hypothesis: what participants really know in the Iowa gambling task. Proc Natl Acad Sci USA 101(45):16075-16080

Maia TV, McClelland JL (2005) The somatic marker hypothesis: still many questions but no answers. Trends Cognit Sci 9(4):162-164

Mason MF, Norton MI, Van Horn JD, Wegner DM, Grafton ST, Macrae CN (2007) Wandering minds: the default network and stimulus-independent thought. Science 315(5810):393-395

Roberts WA, Feeney MC (2009) The comparative study of mental time travel. Trends Cognit Sci 13(6):271-277

Ruffman T (2000) Nonverbal theory of mind: is it important, is it implicit, is it simulation, is it relevant to autism. In Astington JW (ed) Minds in the making: essays in honor of David R. Olson. pp 250-266

Sarnecka BW, Carey S (2008) How counting represents number: what children must learn and when they learn it. Cognition 108(3):662-674

Schacter DL, Addis DR (2007) The cognitive neuroscience of constructive memory: remembering the past and imagining the future. Philos Trans Royal Soc B: Biol Sci 362(1481):773-786

Schacter DL, Addis DR, Buckner RL (2007) Remembering the past to imagine the future: the prospective brain. Nat Rev Neurosci 8(9):657-661

Schweinberger SR, Burton AM (2003) Covert recognition and the neural system for face processing. Cortex 39(1):9-30

Shettleworth SJ (2010) Clever animals and killjoy explanations in comparative psychology. Trends Cognit Sci 14(11):477-481

Spreng RN, Mar RA, Kim AS (2009) The common neural basis of autobiographical memory, prospection, navigation, theory of mind, and the default mode: a quantitative meta-analysis. J Cognit Neurosci 21(3):489-510

Sterelny K (2011) From hominins to humans: how sapiens became behaviourally modern. Philos Trans Royal Soc B: Biol Sci 366(1566):809-822

Stuss DT, Alexander MP (2000) Executive functions and the frontal lobes: a conceptual view. Psychol Res 63(3-4):289-298

Suddendorf T, Corballis MC (2007) The evolution of foresight: what is mental time travel, and is it unique to humans? Beha Brain Sci 30(3):299-312

Suddendorf T, Addis DR, Corballis MC (2009) Mental time travel and the shaping of the human mind. Philos Trans Royal Soc B: Biol Sci 364(1521):1317-1324

Szpunar KK (2010) Episodic future thought an emerging concept. Perspect Psychol Sci 5(2):142-162

Tomb I, Hauser M, Deldin P, Caramazza A (2002) Do somatic markers mediate decisions on the gambling task? Nature Neuroscience 5(11), 1103-1104 (author reply 1104)

Tranel D, Damasio AR (1988a) Knowledge without awareness: an autonomic index of facial recognition in prosopagnosics. Science 228:1453-1454

Tranel D, Damasio AR (1988b) Non-conscious face recognition in patients with face agnosia. Beha Brain Res 30:235-249

Tranel D, Damasio H, Damasio AR (1995) Double dissociation between overt and covert recognition. J Cognit Neurosci 7:425-432

Young AW (1994) Covert recognition. In: Farah M, Ratcligg G (eds) The neuropsychology of high-level vision: collected tutorial essays. Lawrence Erlbaum, Hillsdale, NJ, pp 331-358

Zelazo PD, Frye D (1998) Cognitive complexity and control: II. The development of executive function in childhood. Curr Dir Psychol Sci 7(4):121-126 